\title{
Prolonged measurement of lower oesophageal sphincter function in patients with intestinal metaplasia at the oesophagogastric junction
}

\author{
C Wolf, R Timmer, R Breumelhof, C A Seldenrijk, A J P M Smout
}

\begin{abstract}
Background and aims-It has been shown that gastro-oesophageal reflux plays a role in the pathogenesis of intestinal metaplasia (IM) limited to the oesophagogastric junction (OGJ), similar to the pathogenesis of IM in long segments of columnar lined oesophagus. The aim of this study was to examine lower oesophageal sphincter (LOS) function by means of prolonged recording in patients with IM limited to a normal appearing $O G J$.
\end{abstract}

Patients and methods-Eighteen patients with IM at the OGJ (five females, 13 males; mean age 55.4 years) and 22 patients without IM (nine females, 13 males; mean age 53.9 years) underwent conventional stationary oesophageal manometry. Thereafter, seven hour water perfused manometry with simultaneous pH measurement (probe $5 \mathrm{~cm}$ proximal to the LOS) was performed. Swallowing was monitored with a pharyngeal sidehole and LOS pressure was recorded with a Dent sleeve. Patients were studied in the fasted state (three hours) and after a standardised meal (four hours). LOS pressure was analysed using customised software, and the incidence of reflux episodes ( $\mathrm{pH}<4$ for at least five seconds) and transient LOS relaxations (TLOSRs) were examined. TLOSRs were judged to be accompanied by reflux if a decrease of $1 \mathrm{pH}$ unit occurred during relaxation.

Results-Patients with IM at the OGJ had a higher prevalence of postprandial acid reflux compared with patients without IM. No differences were observed in LOS pressure (pre- and postprandially) or in the prevalence of TLOSRs. However, in the postprandial phase, the rate of TLOSRs accompanied by acid reflux was increased in patients with IM.

Conclusion-Patients with IM at the OGJ have a higher prevalence of postprandial acid reflux. This is not associated with a higher prevalence of TLOSRs or a decreased LOS pressure but with a higher rate of TLOSRs accompanied by reflux. (Gut 2001;49:354-358)

Keywords: intestinal metaplasia; gastro-oesophageal reflux; transient lower oesophageal sphincter relaxations; oesophagogastric junction

While many studies have examined the pathogenesis of traditional columnar lined oesophagus with intestinal metaplasia (IM) (Barrett's oesophagus with a columnar lining $>3 \mathrm{~cm}$ and biopsy proved IM), there are only a few studies which have focused on the pathogenesis of IM in short segments of columnar lining or IM confined to the area of the oesophagogastric junction (OGJ). IM harbours the risk of malignant transformation and adenocarcinomas have also been observed in short columnar lined segments. ${ }^{12}$ Research interest has focused on IM at or close to the OGJ because of the increasing incidence of adenocarcinomas in this area. ${ }^{3}$ It is important to know more about the pathogenesis of IM in this region as it might be possible to treat the underlying mechanism in order to prevent the neoplastic transformation or even to achieve regression of IM. In patients with short segments of columnar lining with IM, a higher incidence of acid gastro-oesophageal reflux has been observed..$^{5-7}$ Even IM limited to an endoscopically normal appearing OGJ has been found to be associated with gastro-oesophageal reflux. ${ }^{8}$ There are no clear data concerning the mechanism underlying reflux in this patient group. A reduction in lower oesophageal sphincter (LOS) pressure in patients with IM has been described. ${ }^{8}$ However, this was measured with conventional stationary manometry, a technique that does not allow prolonged monitoring of LOS function in different digestive states. One important mechanism for gastro-oesophageal reflux is transient lower oesophageal sphincter relaxations (TLOSRs), characterised by an abrupt decrease in LOS pressure that is not associated with swallowing. ${ }^{9-11}$ The increased prevalence of reflux in patients with IM at the OGJ could be due to an increased number of TLOSRs. Therefore, we wished to study the prevalence of TLOSRs and to monitor LOS function over a longer period of time, including the postprandial phase, in patients with IM at an endoscopically normal OGJ compared with patients without IM.

\section{Materials and methods}

STUDY GROUP

The study population consisted of two groups: patients with IM at the OGJ and patients without IM. Patients were recruited from a group

Abbreviations used in this paper: IM, intestinal metaplasia; OGJ, oesophagogastric junction; SCJ, squamocolumnar junction; LOS, lower oesophageal sphincter; TLOSR, transient lower oesophageal sphincter relaxation; SAPLOSR, swallow associated prolonged lower oesophageal sphincter relaxation; MSAPLOSR, multiple swallow associated prolonged lower oesophageal sphincter relaxation; SANLOSR, swallow associated normal lower oesophageal sphincter relaxation; OR, odds ratio. 
who participated in a previous biopsy study performed by our group. In that study all patients had been endoscoped within the past six months and were found to have a normal appearing OGJ. During that endoscopy two forceps biopsies were obtained from immediately distal to the squamocolumnar junction (SCJ) - that is, within $0-5 \mathrm{~mm}$ of the SCJ. Additional biopsies were taken from the distal stomach, antrum, corpus, and "cardia", the latter being defined $2 \mathrm{~cm}$ distal to the proximal stop of the gastric folds. All biopsies were stained with haematoxylin and eosin, alcian blue, and modified Giemsa. The diagnosis of IM was made according to histological findings. Patients were classified as IM positive if goblet cells intensively stained with alcian blue were identified. The IM positive group consisted of 18 patients (five female, 13 male) with a mean age of 55.4 years (SD 10.4); six had a hiatal hernia. The IM negative group consisted of 22 patients (nine female, 13 male; mean age 53.9 (14.9) years; six patients had a hiatal hernia). There were no significant differences between the two groups concerning the use of acid reducing medication, reflux symptoms, smoking habits, and prevalence of Helicobacter pylori infection.

MANOMETRIC AND $\mathrm{pH}$ RECORDING SETUP

After an overnight fast all patients first underwent a conventional stationary manometry. A polyvinyl catheter with three sideholes located $5 \mathrm{~cm}$ apart was introduced nasally. The catheter was connected to a low compliance pneumohydraulic pump and perfused with distilled water at a rate of $0.5 \mathrm{ml} / \mathrm{min}$. A station pull through was performed to locate the LOS and then the oesophageal response to 10 wet swallows $(5 \mathrm{ml})$ was tested. Thereafter the catheter was removed.

A micromanometric silicone catheter was introduced (Dentsleeve Pty Ltd, Parkside, Australia). This 11 lumen catheter had a total length of $160 \mathrm{~cm}$, its external diameter was 3.5 $\mathrm{mm}$, and the internal diameter of the individual channels was $0.4 \mathrm{~mm}$, except for one centrally located infusion channel $(1.9 \mathrm{~mm})$. Four pharyngeal sideholes (located 24, 26, 28, and $30 \mathrm{~cm}$ above the proximal border of the sleeve) were used to monitor swallowing and three sideholes were located in the oesophageal body $(4,9$, and $14 \mathrm{~cm}$ above the sleeve). A reverse perfused $6 \mathrm{~cm}$ long Dent sleeve was used for recording LOS pressure and a sidehole at the proximal border of the sleeve was used for its positioning. Gastric pressure was recorded from a sidehole located $2 \mathrm{~cm}$ below the distal border of the sleeve. The catheter was connected to external pressure transducers (Abbott, Chicago, Illinois, USA) which were mounted on a belt. ${ }^{12}$ A customised water pump (water reservoir $360 \mathrm{ml}$, kept at a pressure of 1 bar) developed by the Department of Biomedical Engineering of the University Medical Centre Utrecht, was used to perfuse the resistors via a silicone manifold (Dentsleeve Pty Ltd). The resistors reduced the water flow to a perfusion rate of $0.08 \mathrm{ml} / \mathrm{min} .{ }^{13}$ The pressure transducers were connected to a portable data logger (MMS, Enschede, the Netherlands) that digitised the data at a rate of 4 or 8 $\mathrm{Hz}$ (oesophageal and pharyngeal recordings, respectively). Patients wore the belt around their waist so that the transducers were located directly distal to the lower end of the ribcage. After correct positioning of the manometry catheter a glass $\mathrm{pH}$ probe was introduced through the same nostril and placed with its tip located $5 \mathrm{~cm}$ above the oral border of the LOS. This probe was also connected to the data logger.

\section{STUDY PROTOCOL}

Patients were studied in a semi recumbent position. After a short accommodation period, data recording was started. Subjects were studied preprandially for three hours and for another four hours after a standardised solid meal (Menu Nasi, Iglo, the Netherlands; total energy content $1575 \mathrm{~kJ}, 45 \mathrm{~g}$ carbohydrate, $12.5 \mathrm{~g}$ fat, $22.5 \mathrm{~g}$ protein). The study was approved by the ethics committee of $\mathrm{St}$ Antonius Hospital.

\section{ANALYSIS OF PRESSURE RECORDINGS}

Data from the portable logger were downloaded to a PC and visualised with commercially available software (MMS, Enschede, the Netherlands). LOS pressure was evaluated using a computer program that was developed by one of the investigators (AJPMS). This program constructs the end expiratory LOS pressure curve and calculates mean end expiratory LOS pressure values (with respect to gastric baseline pressure) for the individual study periods. It has been validated by comparing its results with manual analysis by several experienced investigators (MA van Herwaarden, unpublished data).

The prevalence of TLOSRs was analysed for the pre- and postprandial periods. TLOSRs were defined using established criteria: abrupt decrease $(>0.4 \mathrm{kPa} / 3 \mathrm{~s})$ in end expiratory LOS pressure (nadir pressure of $<0.4 \mathrm{kPa}$ ) not initiated by a swallow (no signal in pharyngeal channels four seconds before or two seconds after the onset of relaxation) with a relaxation duration of at least five seconds. ${ }^{14}{ }^{15}$ During this analysis the $\mathrm{pH}$ channel was hidden from the observer. The prevalence of reflux episodes, defined as an abrupt decrease in $\mathrm{pH}<4$ for at least five seconds, was examined for the preand postprandial study periods. The number of reflux episodes and number of TLOSRs were calculated per hour. The underlying mechanism of reflux events was classified as being one of the following: (1) TLOSR, (2) normal swallow associated relaxation (SANLOSR, pharyngeal peak within four seconds before or two seconds after the onset of relaxation, duration of relaxation shorter than $10 \mathrm{~s}$ ), (3) swallow associated prolonged LOS relaxation (SAPLOSR, pharyngeal peak within four seconds before or two seconds after the onset of relaxation, duration of relaxation equal to or longer than $10 \mathrm{~s}$ ), (4) multiple swallow associated LOS relaxation (MSAPLOSR, multiple pharyngeal peaks within four seconds before or during relaxation), (5) low LOS pressure 


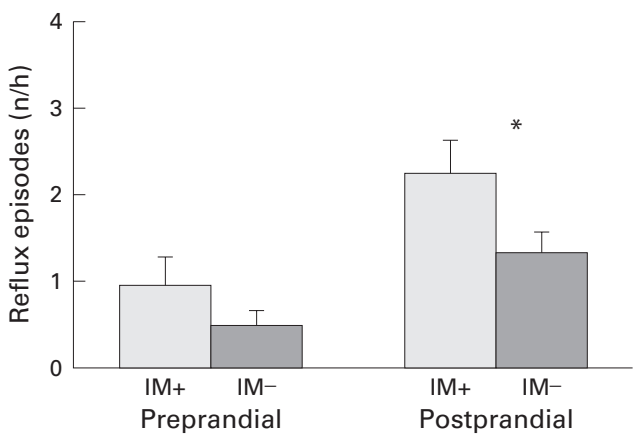

Figure 1 Prevalence of reflux episodes in patients with (+) and without (-) intestinal metaplasia (IM) in the pre- and postprandial periods (mean (SEM)). ${ }^{\star} p=0.042$, IM positive versus IM negative group.

(undetectable LOS pressure for at least $30 \mathrm{~s}$ before reflux), or (6) straining (sharp and brief positive elevations in gastric and oesophageal pressure prior to $\mathrm{pH}$ decrease). Reflux was judged to have accompanied a TLOSR if a decrease in oesophageal $\mathrm{pH}$ greater than $1 \mathrm{pH}$ unit was observed during the relaxation.

STATISTICAL ANALYSIS

Data are expressed as mean (SEM) or percentages. Statistical analysis was performed with SPSS software. The $\chi^{2}$ test, Mann-Whitney U test, and $t$ test were used where appropriate.

\section{Results}

As shown in fig 1, the prevalence of reflux episodes was higher in the postprandial than in the fasting period $(0.7 /$ hour and 1.8 /hour, respectively; odds ratio (OR) 2.08; 95\% confidence interval (CI) 1.33-3.26). Patients with IM at the OGJ had more reflux episodes per hour in the postprandial state compared with patients without IM (2.25/hour $v 1.35$ /hour, respectively; OR 1.65; 95\% CI 1.01-2.74). Similar findings were noted for the percentage of time with $\mathrm{pH}$ below 4 in the postprandial phase (1.9\% v 4.38\%; OR 1.42 ; 95\% CI $1.03-1.95)$ (table 1). There was no difference between the two groups in the preprandial phase. End expiratory LOS pressures did not differ between the two groups neither preprandially or in the postprandial phase (table 1). If both groups were analysed together a trend towards a lower LOS pressure in the postprandial state was observed (1.12 and $0.96 \mathrm{kPa}$; ns). For the entire group, the prevalence of TLOSRs (calculated per hour) was higher in the postprandial period than in the preprandial period (1.8/hour and 2.9/hour, respectively; OR 2.4 ; 95\% CI 1.48-3.88). The prevalence of TLOSRs pre- and postprandially was not different between IM positive and IM negative

Table 1 Reflux parameters and lower oesophageal sphinter (LOS) characteristics in patients with and without intestinal metaplasia (IM) at the oesophagogastric junction

\begin{tabular}{llll}
\hline & $I M+(n=18)$ & $I M-(n=22)$ & OR $(95 \% C I)$ \\
\hline \% time pH <4 & & & \\
$\quad$ Preprandial period (\%) & $1.75(0.46)$ & $1.15(0.22)$ & $1.33(0.84-2.10)$ \\
$\quad$ Postprandial period (\%) & $4.38(0.99)$ & $1.90(0.34)$ & $1.42(1.03-1.95)$ \\
End expiratory LOS pressure & & & $0.76(0.24-2.48)$ \\
$\quad$ Preprandial period (kPa) & $1.10(0.09)$ & $1.18(0.14)$ & $0.55(0.14-2.10)$ \\
\hline Postprandial period (kPa) & $0.94(0.09)$ & $1.09(0.13)$ & \\
\hline
\end{tabular}

Data are mean (SEM) with odds ratios (OR) and 95\% confidence interval (CI).

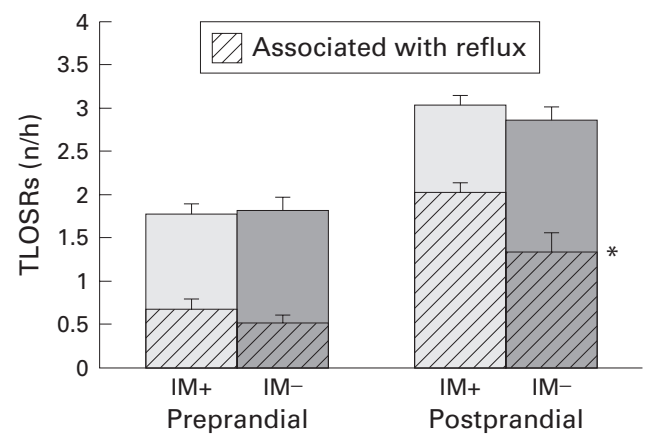

Figure 2 Prevalence of transient lower oesophageal sphincter relaxations (TLOSR) s and TLOSRs associated with reflux in patients with (+) and without (-) intestinal metaplasia (IM) (mean (SEM)). ${ }^{\star} p=0.05$, TLOSRs associated with reflux, IM positive versus IM negative group.

patients. However, a higher rate of TLOSRs accompanied by reflux (postprandially) was observed in patients with IM at the OGJ. In these patients $66.9 \%$ of TLOSRs were associated with reflux compared with $46.5 \%$ in patients without IM (OR $7.41 ; 95 \%$ CI 1.02-60.58) (fig 2). Pre- and postprandially there was no difference in the prevalence of SAPLOSRs or MSAPLOSRs, or in the rate of SAPLOSRs or MSAPLOSRs associated with reflux between patients with or without IM.

Preprandially, patients with IM had a total number of 49 reflux episodes compared with 34 reflux episodes in IM negative patients. As shown in fig 3, the mechanisms through which these reflux episodes occurred were not different between groups (IM+ 57.1\% TLOSRs, $4.1 \%$ SAPLOSRs, $4.1 \%$ MSAPLOSRs, $22.5 \%$ SANLOSRs, 0\% low LOS pressure, $12.2 \%$ straining; IM- $58.8 \%$ TLOSRs, $2.9 \%$ SAPLOSRs, $\quad 0 \%$ MSAPLOSRs, $\quad 23.5 \%$ SANLOSRs, $2.9 \%$ low LOS pressure, $11.7 \%$ straining). In the postprandial period a total of 135 reflux episodes occurred in the IM positive patients $(57.1 \%$ TLOSRs, $5.9 \%$ SAPLOSRs, $5.2 \%$ MSAPLOSRs, $13.3 \%$ SANLOSRs, $6.7 \%$ low LOS pressure, $11.9 \%$ straining) and a total of 97 episodes in the IM negative patients $(70.1 \%$ TLOSRs, $6.2 \%$ SAPLOSRs, $4.1 \%$ MSAPLOSRs, $12.4 \%$ SANLOSRs, $2.1 \%$ low LOS pressure, $5.2 \%$ straining).

\section{Discussion}

Barrett's oesophagus is characterised by the presence of columnar lining with IM in the distal oesophagus. It is known that this condition is the consequence of longstanding gastro-oesophageal reflux disease caused by disturbances in oesophageal motility and LOS dysfunction. ${ }^{16-18}$ Patients with Barrett's oesophagus have a 30-40-fold increased risk of developing oesophageal malignancies. ${ }^{19}$ IM in the columnar lining of Barrett's oesophagus represents a precursor lesion for oesophageal adenocarcinoma. ${ }^{2}$ As the prevalence of carcinomas in the area of the OGJ has been increasing over the last 10 years, IM in that region and its pathogenesis have gained research interest. ${ }^{34}$ In an earlier study (unpublished data) we confirmed the results of Öberg et al in a similar patient population. ${ }^{8}$ The results of our and 


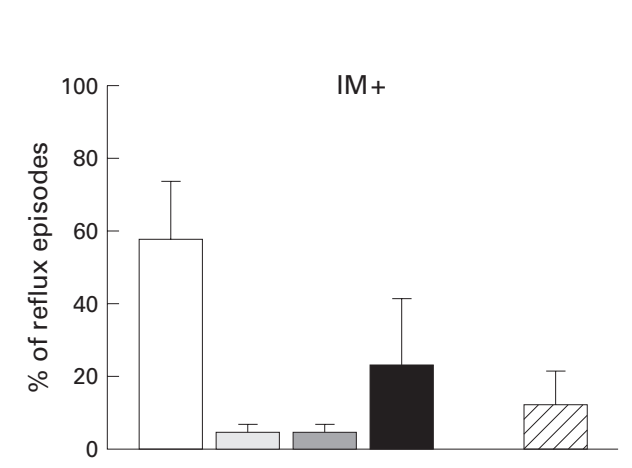

Preprandial
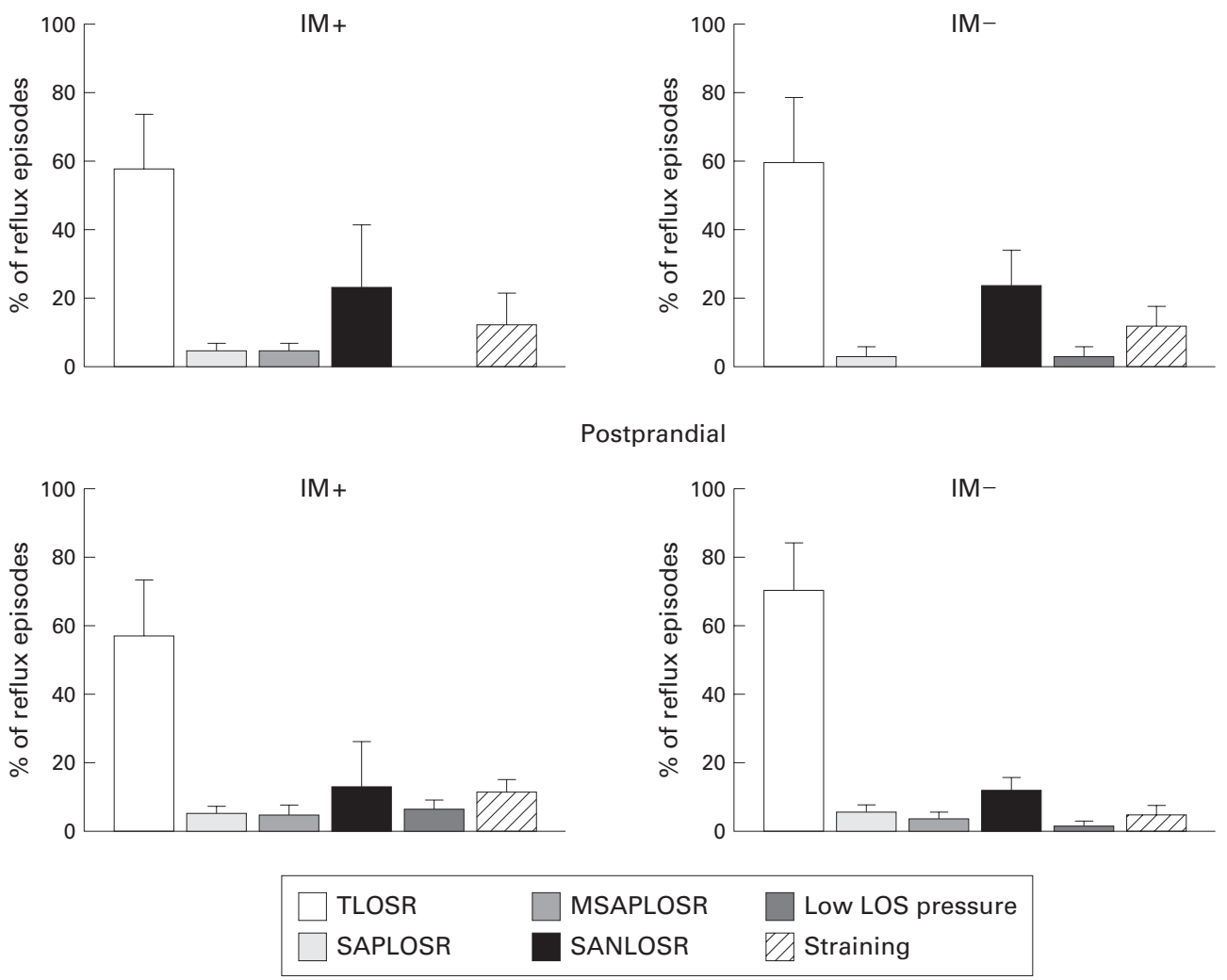

Figure 3 Distribution of reflux mechanisms (mean (SEM), expressed as percentage of reflux episodes) in patients with $(+)$ and without $(-)$ intestinal metaplasia (IM), pre- and postprandially. No significant differences were found. LOS, lower oesophageal sphincter; TLOSR, transient lower oesophageal sphincter relaxation; SAPLOSR, swallow associated sphincter relaxation; SANLOSR, swallow associated normal lower oesophageal sphincter relaxation.

their studies suggest a causative role for gastrooesophageal reflux disease, similar to long segment traditional Barrett's oesophagus. However, in contrast with Öberg et al, we could not find a clear reduction in LOS competence, as measured with short term conventional manometry. In the interpretation of results obtained from stationary oesophageal manometry, one has to bear in mind that the LOS is not a constant high pressure zone but a dynamic structure that undergoes physiological changes in the circadian rhythm in healthy subjects as well as in patients with gastrooesophageal reflux disease. ${ }^{9} 1020$ Therefore, LOS measurements that monitor LOS function over a longer period of time provide more information than short term recordings. Prolonged recordings of LOS function can be obtained with a miniature Dent sleeve assembly. Oesophageal body and sphincter measurements with this device have been shown to be reproducible and stable. ${ }^{21}$ The feasibility of the recording system that we used (transducers on belt, portable perfusion pump) has been demonstrated previously. ${ }^{12}{ }^{13}$

To our knowledge this is the first study that has measured LOS function over a prolonged period of time in patients with IM at the OGJ and in a comparable group of patients without IM. This method not only allows measurement of LOS pressure but it is also possible to assess LOS relaxation. Low LOS pressure and TLOSRs have been shown to be important factors in the pathogenesis of gastrooesophageal reflux. Simultaneous $\mathrm{pH}$ recording in the distal oesophagus permits identification of the underlying mechanisms of observed reflux episodes.

Results obtained with this new method support the earlier findings that gastrooesophageal reflux plays a role, as we found a higher prevalence of reflux episodes in patients with IM compared with patients without IM. This higher prevalence was observed in the postprandial state only. We did not find a statistically significant difference in the prevalence of LOS pressure or TLOSRs between the two groups, either in the preprandial or postprandial state. However, we observed that in the postprandial period the proportion of TLOSRs associated with reflux was higher in patients with IM. These findings indicate that the increased oesophageal acid exposure in IM positive patients is caused by factors other than the "traditional" reflux mechanisms such as low LOS pressure and a high prevalence of TLOSRs. The prevalence of a hiatal hernia was not different between the two groups and therefore it seems unlikely that this played a role in the current study. Apparently, some other factor is involved in patients with IM at the OGJ which causes relaxations of the LOS to lead more readily to reflux than in patients without IM. The most likely explanation for the observed phenomenon is that in patients with IM, the volume, acidity, or distribution of 
gastric contents favours reflux more than in IM negative patients. This could be due to a slower rate of gastric emptying, a higher rate of gastric acid secretion, altered intragastric content distribution, or differences in postprandial gastric motility. ${ }^{22-26}$

In conclusion, using a method for prolonged monitoring of LOS function with combined $\mathrm{pH}$ measurement, this study provided evidence that IM at an endoscopically normal OGJ was associated with acid gastro-oesophageal reflux. LOS pressure was not lower in patients with IM compared with patients without IM. Furthermore, a higher prevalence of TLOSRs was not observed in the IM positive patient group. However, these patients had a higher prevalence of postprandial acid gastrooesophageal reflux; the percentage of TLOSRs associated with acid reflux was increased. Elucidation of mechanisms underlying this requires further studies.

We would like to thank the endoscopy and pathology departments for their support in the study. This study was supported by Aventis BV, the Netherlands.

1 Schnell TG, Sontag SJ, Chejfec G. Adenocarcinomas arising in tongues or short segments of Barrett's esophagus. Dig Dis in tongues or short se

2 Haggitt RC. Barrett's esophagus, dysplasia, and adenocarcinoma. Hum Pathol 1994;25: 982-93.

3 Blot WJ, Devesa SS, Kneller RW, et al. Rising incidence of adenocarcinoma of the esophagus and gastric cardia. ҰAMA 1991;265:1287-9.

4 Pera M, Cameron AJ, Trastek VF, et al. Increasing incidence of adenocarcinoma of the esophagus and esophagogastric junction. Gastroenterology 1993;104:510-13.

5 Loughney T, Maydonovitch CL, Wong RKH. Esophageal manometry and ambulatory 24-hour $\mathrm{pH}$ monitoring in patients with short and long segment Barrett's esophagus. Am $\mathcal{7}$ Gastroenterol 1998;93:916-19.

6 Clark GWB, Ireland AP, Peters JH, et al. Short-segment Barrett's esophagus: a prevalent complication of gastroesophageal reflux disease with malignant potential. $\mathcal{F}$ esophageal reflux disease with
Gastrointest Surg 1997;1:113-22.

7 Öberg S, Ritter MP, Crookes PF, et al. Gastroesophageal reflux disease and mucosal injury with emphasis on shortsegment Barrett's esophagus and duodenogastroesophasegment Barrett's esophagus and duodenog

8 Oberg S, Peters JH, DeMeester TR, et al. Inflammation and Oberg $\mathrm{S}$, Peters $\mathrm{JH}, \mathrm{DeMe}$ ester TR, et al. Inflammation and
specialized intestinal metaplasia of cardiac mucosa is a manifestation of gastroesophageal reflux disease. Ann Surg 1997;226:522-32.

9 Dent J, Dodds WJ, Friedman RH, et al. Mechanism of gasroesophageal reflux in recumbent asymptomatic human subjects. $\mathcal{F}$ Clin Invest 1980;65:256-67.

10 Dodds WJ, Dent J, Hogan WJ, et al. Mechanisms of gastroesophageal reflux in patients with reflux esophagitis. $N$ Engl 7 Med 1982;307:1547-52.

11 Mittal RK, Holloway RH, Penagini R, et al. Transient lower esophageal sphincter relaxation. Gastroenterology 1995;109: $601-10$

12 Samsom M, Smout AJ, Hebbard G, et al. A novel portable perfused manometric system for recording of small bowel motility. Neurogastroenterol Motil 1998;10:139-48.

13 van Herwaarden MA, Samsom M, Smout AJPM. 24-h ambulatory LOS pressure recording using a portable water-perfused manometric system and fully automated signal analysis. Neurogastroenterol Motil 1998;10:A72.

14 Holloway RH, Kocyan P, Dent P. Provocation of transient lower esophageal sphincter relaxations by meals in patients with symptomatic gastroesophageal reflux. Dig Dis Sci 1991;36:1034-9.

15 Holloway RF, Penagini R, Ireland AC. Criteria for objective definition of transient lower esophageal sphincter relaxation. Am f Physiol 1995;268:G128-33.

16 Stein JH, Hoeft S, DeMeester TR. Functional foregut abnormalities in Barrett's esophagus. F Thorac Cardiovasc Surg 1993;105:107-11.

17 Singh P, Taylor RH, Colin-Jones DG. Esophageal motor dysfunction and acid exposure in reflux esophagitis are more severe if Barrett's metaplasia is present. Am $\mathcal{F}$ Gastroenterol 1994;89:349-56.

18 Coenraad M, Masclee AAM, Straathof JWA, et al. Is Barrett's esophagus characterized by more pronounced acid reflux than severe esophagitis? Am $\mathcal{f}$ Gastroenterol 1998;93:1068-72.

19 Reid BJ. Barrett's esophagus and esophageal adenocarcinoma. Gastroenterol Clin North Am 1991;20:817-34.

20 Schoeman MN, Tippett MD, Akkermans LMA, et al. Mechanisms of gastroesophageal reflux in ambulant healthy human subjects. Gastroenterology 1995;108:83-91.

21 Crowell MD, Schettler-Duncan VA, Yu SS. Manometric evaluation of esophageal function utilizing a miniature Dent sleeve: within and between session reproducibility in healthy controls. Gastroenterology 1999;115:G4257.

22 McCallum RW, Berkowitz DM, Lerner E. Gastric emptying in patients with gastroesophageal reflux. Gastroenterology 1981;80:285-91.

23 Cadiot G, Bruhat A, Rigaud D, et al. Multivariate analysis of pathophysiological factors in reflux oesophagitis. Gut 1997;40:167-74

24 Vaezi MF, Richter JE. Role of acid and duodenogastric esophageal reflux in GER. Gastroenterology 1996;111: 1192-9.

25 Shay SS, Conwell DL, Mehindru V, et al. The effect of posture on gastroesophageal reflux event frequency and composition during fasting. Am f Gastroenterol 1996;91:54-60.

26 Penagini R, Hebbard G, Horowitz M, et al. Motor function of the proximal stomach and visceral perception in gastrooesophageal reflux disease. Gut 1998;42:251-7. 